\title{
The Novel Monocot-Specific 9-Lipoxygenase ZmLOX12 Is Required to Mount an Effective Jasmonate-Mediated Defense Against Fusarium verticillioides in Maize
}

\author{
Shawn A. Christensen,, ${ }^{1,2}$ Andriy Nemchenko, ${ }^{1,3}$ Yong-Soon Park, ${ }^{1,4}$ Eli Borrego, ${ }^{1}$ Pei-Cheng Huang, ${ }^{1}$ \\ Eric A. Schmelz, ${ }^{2}$ Susan Kunze, ${ }^{5}$ Ivo Feussner, ${ }^{5}$ Nasser Yalpani, ${ }^{6}$ Robert Meeley, ${ }^{6}$ and \\ Michael V. Kolomiets ${ }^{1}$ \\ ${ }^{1}$ Department of Plant Pathology and Microbiology, Texas A\&M University, College Station, TX 77843-2132, U.S.A.; \\ ${ }^{2}$ Chemistry Unit, Center of Medical, Agricultural, and Veterinary Entomology, U.S. Department of Agriculture, Gainesville, \\ FL 32608, U.S.A.; ${ }^{3}$ University of Texas Southwestern Medical Center at Dallas, 5323 Harry Hines Blvd, Dallas, TX 75390, \\ U.S.A.; ${ }^{4}$ Superbacteria Research Center, KRIBB, Daejeon 305-806, South Korea; ${ }^{5}$ Department of Plant Biochemistry, \\ Albrecht-von-Haller-Institute for Plant Sciences, Georg-August University Göttingen, Justus-von-Liebig-Weg 11, D37077 \\ Göttingen, Germany; ${ }^{6}$ DuPont Pioneer, 7300 NW 62nd Ave., Johnston, IA 50131-0552, U.S.A.
}

Submitted 27 June 2014. Accepted 14 July 2014.

Fusarium verticillioides is a major limiting factor for maize production due to ear and stalk rot and the contamination of seed with the carcinogenic mycotoxin fumonisin. While lipoxygenase (LOX)-derived oxylipins have been implicated in defense against diverse pathogens, their function in maize resistance against $F$. verticillioides is poorly understood. Here, we functionally characterized a novel maize 9-LOX gene, $\mathrm{ZmLOX12}$. This gene is distantly related to known dicot LOX genes, with closest homologs found exclusively in other monocot species. $\mathrm{ZmLOX12}$ is predominantly expressed in mesocotyls in which it is strongly induced in response to $F$. verticillioides infection. The Mutator transposon-insertional lox12-1 mutant is more susceptible to $F$. verticillioides colonization of mesocotyls, stalks, and kernels. The infected mutant kernels accumulate a significantly greater amount of the mycotoxin fumonisin. Reduced resistance to the pathogen is accompanied by diminished levels of the jasmonic acid (JA) precursor 12-oxo phytodienoic acid, JA-isoleucine, and expression of jasmonate-biosynthetic genes. Supporting the strong defense role of jasmonates, the JA-deficient opr7 opr8 double mutant displayed complete lack of immunity to $F$. verticillioides. Unexpectedly, the more susceptible lox 12 mutant accumulated higher levels of kauralexins, suggesting that $\boldsymbol{F}$. verticillioides is tolerant to this group of

\section{S. A. Christensen and A. Nemchenko contributed equally to this study.}

Novel materials described in this publication may be available for noncommercial research purposes upon acceptance and signing of a material transfer agreement. In some cases, such materials may contain or be derived from materials obtained from a third party. In such cases, distribution of material will be subject to the requisite permission from any third-party owners, licensors, or controllers of all or parts of the material. Obtaining any permission will be the sole responsibility of the requestor.

Corresponding author: M. V. Kolomiets; Telephone: +1.979.458.4624; Fax: +1.979.845.6483; E-mail: kolomiets@tamu.edu

*The $\boldsymbol{e}$-Xtra logo stands for "electronic extra" and indicates that four supplementary figures are published online.

(c) 2014 The American Phytopathological Society antimicrobial phytoalexins. This study demonstrates that this unique monocot-specific 9-LOX plays a key role in defense against $F$. verticillioides in diverse maize tissues and provides genetic evidence that $\mathrm{JA}$ is the major defense hormone against this pathogen.

Fusarium verticillioides is an ascomycete fungal pathogen that causes ear and stalk rots in maize (Zea mays L.), producing large yield losses in many parts of the world (Desjardins 2003; Rheeder et al. 2002). F. verticillioides is most frequently found in kernels, in which it reduces grain quality by producing fumonisins - a family of carcinogenic mycotoxins responsible for causing deleterious diseases, such as leukoencephalomalacia in horses (Kellerman et al. 1990), pulmonary edema in pigs (Colvin and Harrison 1992), and esophageal cancer in humans (Yoshizawa et al. 1994).

The most common route of $F$. verticillioides invasion is through the silks, but typically, only a minor portion of infected kernels become symptomatic (Munkvold et al. 1997; Oren et al. 2003). Another well-documented pathway for $F$. verticillioides infection is through the underground organs where abundant inoculum spreads to the roots and through the mesocotyls to the stalk and the ears (Munkvold et al. 1997; Oren et al. 2003). Systemic infection by F. verticillioides, especially at early disease stages, may result in blight of seedlings as well as root and stalk rots (Nelson 1992). At least in some maize inbred lines, transmission of the fungus from the seedling mesocotyl and crown to the stalk is a limiting step of infection (Munkvold et al. 1997). Etiolated maize mesocotyls were demonstrated to be an effective tool to investigate maizefungus interactions including $F$. verticillioides (Heim et al. 1983; Oren et al. 2003).

The infection process of maize tissue is influenced by a number of environmental and host biochemical factors. Among the latter, a group of oxidized lipid-derived molecules termed oxylipins act as signals to modulate reproductive development, germination, senescence, programmed cell death, tolerance to cold, drought, and salt stresses, plant-to-plant and plant-to-pest communication, and resistance to pathogens (Feussner and Wasternack 2002; Howe and Jander 2008; Shah 
2005). Most plant oxylipins identified to date are formed via enzymatic activity of the lipoxygenase (LOX; EC 1.13.11.12) pathway. LOX catalyzes the oxygenation of both linoleic (18:2) and linolenic (18:3) polyunsaturated fatty acids, at either carbon position 9 or 13 in the fatty acid backbone, thereby resulting in the formation of distinct 9- and 13-hydroperoxides, respectively. Thus, based on the position of carbons in the fatty acid chain targeted for oxygenation, LOX are referred to as 9or 13-LOX (Liavonchanka and Feussner 2006).

While several studies have shown that 9-LOX-derived oxylipins have chemical properties that exhibit antimicrobial activity (Prost et al. 2005; Weber et al. 1999), a genetic investigation into the physiological function of $9-\mathrm{LOX}$ in pathogen defense has only recently begun. In tobacco (Nicotiana tobacum), NtLOX1 antisense plants have reduced levels of 9LOX-derived divinyl ethers and are impaired in resistance to Phytophthora parasitica (Fammartino et al. 2007; Rance et al. 1998). In pepper (Capsicum annuum), silencing of a 9-LOX, CaLOX1, led to increased susceptibility to Xanthomonas campestris pv. vesicatoria and Colletotrichum coccodes infection (Hwang and Hwang 2010). In Arabidopsis, mutation of the CaLOX1 ortholog AtLOX1 diminished resistance to a variety of Solanaceous pathogens (Hwang and Hwang 2010) and genetically showed that AtLOX1 regulates both local and systemic defense responses to Pseudomonas syringae pv. tomato via 9-ketooctadecatrienoic acid (9-KOTE) (Vicente et al.



Fig. 1. Sequence analysis of the $Z m L O X 12$ gene. A, Southern blot analysis revealed the presence of genes homologous to $Z m L O X 12$ in other monocot species. Genomic DNA extracted from the maize line B73 (Zea mays L.), sorghum (Sorghum bicolor. cv. B1), rice (Oryza sativa cv. Cutter), and millet (Panicum miliaceum L) was digested with XbaI, separated on the electrophoresis gel, blotted onto nylon membranes, and was hybridized to the original expressed sequence tag clone p0006.camah52, a nearly fulllength cDNA sequence lacking 24 bp after the start codon. DNA size markers in kilobases $(\mathrm{kb})$ are indicated on the left.
2012). Simultaneous disruption of the only 9-LOX in Arabidopsis AtLOX1 and AtLOX5 demonstrated that 9-hydroxy10,12,15-octadecatrienoic acid (9-HOTE) antagonizes ethylene responses yet positively modulates oxidative stress, lipid peroxidation, and plant defense (Lopez et al. 2011). While these recent genetic studies have helped to elucidate the function of 9LOX in pathogen defense for dicot species, there is little information on the role of 9-LOX in monocots, especially in response to the agronomically important mycotoxin-producing seed pathogen $F$. verticillioides.

Towards this objective, we recently characterized maize plants harboring a Mutator $(M u)$ transposon in the 9-LOX gene $\mathrm{ZmLOX3}$ (Gao et al. 2007). Although 9-oxylipins are generally believed to have defensive roles, surprisingly, lox3 mutants proved highly resistant to $F$. verticillioides infection in stalks and had greatly reduced levels of fumonisin $B_{1}$ in kernels. Thus, some 9-LOX facilitate fungal pathogenicity and, therefore, contribute to susceptibility. While these findings further confirm the notion that pathogens manipulate plant lipid derivatives to facilitate pathogenesis (Christensen and Kolomiets 2011), there remains a need to identify one or more maize 9LOX genes that are involved in resistance to pathogens. In our screen for LOX genes that mediate maize resistance to $F$. verticillioides, we discovered a unique monocot-specific 9-LOX,


Interestingly, recent analysis of the genetic diversity in the maize LOX gene family showed that this gene, unlike any other maize LOX, has been under stringent selection since the domestication of maize (De La Fuente et al. 2013). Disruption of ZmLOX12 caused a significant decrease in mesocotyl resistance to $F$. verticillioides, which was accompanied by lower levels of key jasmonates, including 12-oxo phytodienoic acid (12OPDA) and jasmonic acid-isoleucine (JA-Ile). In kernel bioassays, lox 12 mutants were heavily colonized by $F$. verticillioides and accumulated greater levels of fumonisin $\mathrm{B}_{1}$ than their respective wild types. Stalks also had higher incidence and severity of disease in lox 12 mutants, indicating a broad defense function for $\mathrm{ZmLOX} 12$ in a range of maize organs. Accordingly, the results of this study show that the maize 9-LOX isoform, $\mathrm{ZmLOX12}$, plays a fundamental role in resistance to F. verticillioides.

\section{RESULTS}

Cloning and sequence analysis of the $\mathrm{ZmLOX12}$ gene.

Among approximately 800,000 expressed sequence tags (EST) representing more than 260 diverse cDNA libraries available at the extensive Pioneer and DuPont databases, only two of the 767 EST clones encoding maize LOX genes were representative of $\mathrm{ZmLOX12}$ (P0105.camah52r and $P 0126 . c n l d n 76 r)$. This suggests that $Z m L O X 12$ is expressed at a relatively low level in most healthy maize tissues. The sequence from the longest EST clone (P0105.camah52r) represents almost the entire coding sequence of the gene, missing only $24 \mathrm{bp}$ of the $5^{\prime}$ coding sequence. The remaining portion along with the $5^{\prime}$ untranslated region sequence was cloned by a $5^{\prime}$ rapid amplification of cDNA ends technique from a root cDNA library. The combined full-length cDNA sequence contained a complete open reading frame of 2,550 bp and was named $Z m L O X 12$ (GenBank accession number DQ335770). The predicted protein sequence encodes an 850 amino acid peptide with the estimated molecular mass of 96 $\mathrm{kDa}$ and isoelecric point of 8.1. The deduced amino acid sequence of ZmLOX12 exhibited the highest homology (57\%) to the translated rice cDNA AK064795 (Bachmann et al. 2002) but only between 39 and $45 \%$ identity to the rest of the maize LOX and less than $40 \%$ to any other reported LOX from 
other monocot or dicot species. This low sequence identity to other plant LOX underscores the uniqueness of ZmLOX12 and warranted further molecular and biochemical characterization.

To examine whether a gene closely related to $Z m L O X 12$ is present in maize or other monocot species, we used Southern blot analysis with the entire insert from the EST clone P0105.camah52r as a probe. In the maize B73 inbred line, only a single band was identified, suggesting that a single copy of $\mathrm{ZmLOX} 12$ is present in the maize genome (Fig. 1). A single copy was also found in rice; in sorghum however, the ZmLOX12 probe hybridized to two bands with similar intensity, suggesting the presence of two ZmLOX12-like sequences. Indeed, BLAST search of the sorghum genome sequences available at the Sorghum Genome Project website revealed the existence of two highly similar but distinct contig sequences, super_184 and super_41, that shared, respectively, 88 and $87 \%$ amino acid sequence identity with ZmLOX12. In addition to sorghum and rice, the proso (millet) genome appears to encode a $Z m L O X 12$ like sequence, although it shares less homology to $Z m L O X 12$, as evidenced by a less-intense hybridization signal (Fig. 1). Further BLAST comparisons of the ZmLOX12 gene against available genome sequences of dicot species yielded no $\mathrm{ZmLOX12-like}$ sequences, suggesting that $Z m L O X 12$ is a monocot-specific LOX.

To further characterize $\mathrm{ZmLOX} 12$, we analyzed the deduced amino acid sequence. Analysis of the sequence domain architecture showed that the protein comprised two major domains: the lipoxygenase domain, typical for all LOX, and the PLAT domain, known to mediate membrane attachment via other protein-binding partners (Marchler-Bauer et al. 2005). A close proximity to the membrane lipids may help ensure constant availability of fatty acid substrates. Subcellular localization of ZmLOX12 is under current investigation.

Additional analysis of the ZmLOX12 deduced amino acid sequence revealed that, like other known plant LOX, ZmLOX12 harbors all five canonical residues involved in iron binding and enzyme catalytic activity (Prigge et al. 1996): His532, His-541, His-699, Asn-703, and Ile-850. Similar to other LOX, ZmLOX12 possesses motifs required for substrate (AW RSDEEFAREMLAG) and oxygen binding (ASALHAAVNFG QY) (Santino et al. 2005), in positions 355 to 369 and 695 to 707, respectively. As a unique feature of ZmLOX12, instead of the common conventional motif $\mathrm{Thr} / \mathrm{Val}$ reported to be indicative of all plant 9-LOX known to date (Hornung et al. 1999; Liavonchanka and Feussner 2006), ZmLOX12 contains Thr/Met (566/567), which distinguishes it as a novel LOX.

\section{Biochemical properties of recombinant $\mathrm{ZmLOX12}$.}

It was difficult to predict accurately whether ZmLOX12 is a 9- or 13-LOX based solely on sequence data. Therefore, enzymatic properties of ZmLOX12 were examined by overexpressing the recombinant protein in Escherichia coli under the control of an isopropyl thio- $\beta$-D-galactopyranoside (IPTG)-inducible promoter. ZmLOX12 was highly active at neutral to slightly acidic $\mathrm{pH}$, with maximal LOX activity observed at $\mathrm{pH} 6.0$ (Fig. 2). Regiospecificity of the enzyme was determined at $\mathrm{pH}$ 6.0 and $\mathrm{pH}$ 8.0, using linoleic acid as a substrate (Fig. 2). Greater than $90 \%$ of ZmLOX12-catalyzed products were $9 S$ hydroperoxides; thus, we conclude that ZmLOX12 displays predominant 9-LOX regiospecificity. With linoleic acid as a substrate, the preferred products of ZmLOX12 enzymatic activity, detected by high-performance liquid chromatography, were hydro(pero)xy diene $(9 S, 10 E, 12 Z)$-9-hydro(pero)xy10,12-octadecadienoic acid (9-H(P)ODE) and keto diene (10E,12Z)-9-keto-10,12-octadecadienoic acid (9-KODE) (Fig. 2 ). The fact that the resultant oxygenated fatty acids were predominantly $S$-enantiomers strongly suggests that they are not a product of autocatalysis but, rather, a result of enzymatic conversion (Mueller et al. 2006). Among the 9-LOX products, 9-HODE was preferentially produced $(92 \%)$ as compared with 9-KODE $(6.25 \%)$, whereas only minor quantities of $13-\mathrm{LOX}$ products were made as a racemic mixture of $S$-and $R$-enantiomers. As a control, we used the maize 13-LOX gene ZmLOX10, which was clearly identified as a 13-LOX by Nemchenko and associates (2006). This negative control showed no detectable 9-LOX activity (data not shown). Collectively, these data suggest that ZmLOX12 is a linoleate 9-LOX that is active within a $\mathrm{pH}$ range encompassing natural cytosol conditions.

\section{Generation of lox12-1 mutant} and wild-type near-isogenic lines.

For analysis of $\mathrm{ZmLOX} 12$ gene architecture, full-length cDNA was aligned with the corresponding genomic sequence derived from the available maize genome. This analysis revealed that the $Z m L O X 12$ gene is comprised of six exons and five introns (Fig. 3A). To search for mutations in the ZmLOX12 gene, a transposon insertional reverse genetic approach was used. $Z m L O X 12$-specific and $M u$ terminal inverted repeat-specific primers were used to screen approximately 42,000 $\mathrm{Mu}$ insertional individual plants available at Dupont Pioneer (Johnston, IA, U.S.A.). This large population contains $\mathrm{Mu}$ insertions throughout the maize genome and allows efficient identification of mutants for the majority of maize genes (McCarty and Meeley 2009). Polymerase chain reaction (PCR)-based screening of this population resulted in the identification of a single mutant allele named lox $12-1$. Sequence analysis of the adjoining sequences on both sides of the insertion showed that lox 12-1 had a $M u$ element inserted in exon VI, 23 bp upstream of the stop codon (Fig. 3A). This $3^{\prime}$ end of the $\mathrm{ZmLOX} 12$ gene encodes a C-terminus region that is highly conserved across all maize LOX (Fig. 3B) and LOX from other dicot and monocot plant species (Supplementary Fig. $\mathrm{S} 1$ ), suggesting the requirement of this conserved $\mathrm{C}$ domain for LOX functionality. To identify homozygous wild type (WT), mutant, and heterozygous plants in a segregating population, Southern blot analysis was used. Figure 3C shows that both WT and homozygous mutant individuals displayed a single band that hybridized to the ZmLOX12 probe. However, the mutant band was approximately $1.5 \mathrm{~kb}$ larger than that of the WT allele, a difference expected from an insertion of an aver-

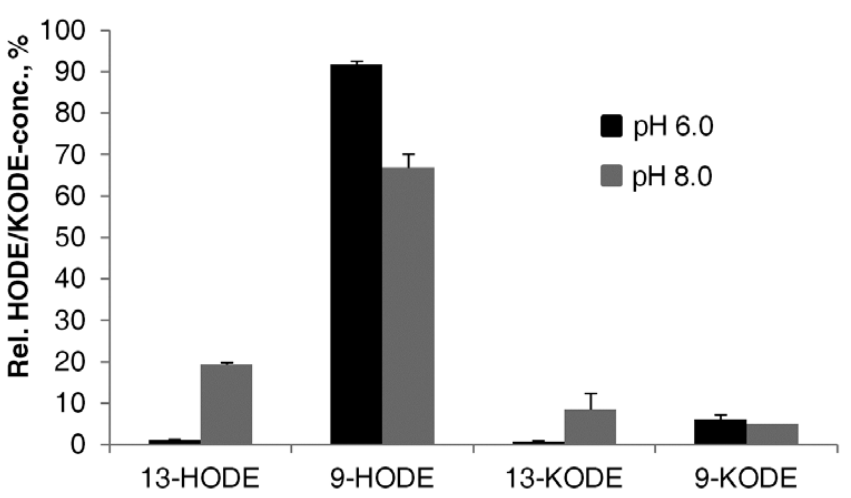

Fig. 2. Regiospecificity analysis of the recombinant $\mathrm{ZmLOX} 12$ protein. Analysis of lipoxygenase (LOX) regiospecificity at pH-values 6.0 and 8.0 is reflected in conversion of linoleic acid by the recombinant $\mathrm{ZmLOX} 12$ into correspondent 13-HODE, $(9 Z, 11 E, 13 S)$-13-hydroxy-octadeca-9,11dienoic acid; 9-HODE, (9S,10E,12Z)-9-hydroxy-10,12-octadecadienoic acid; 13-KODE, (9Z,11E)-13-oxo-octadeca-9,11-dienoic acid; and 9-KODE, ketodiene $(9 S, 10 E, 12 Z)$-9-oxo-10,12-octadecadienoic acid. Relative concentration of LOX products ( $y$ axis) is presented as a portion of the sum of all isomers which was set as $100 \%$. 
age $M u$ element in the gene. As expected, heterozygous individuals displayed both the WT and mutant bands. To test for the presence of the ZmLOX12 transcript in lox12-1 mutant, total RNA was extracted from mesocotyls of both WT and homozygous mutant individuals and $\mathrm{ZmLOX} 12$ transcripts were measured. Semiquantitative reverse transcription (RT)-PCR using $Z m L O X 12$ gene-specific primers confirmed the absence of the ZmLOX12 transcripts in the mesocotyls of homozygous mutant lox12-1 plants (Fig. 3D). To eliminate unrelated mutations, the lox12-1 allele was backcrossed four times into the B73 genetic background, resulting in the generation of nearisogenic mutant and WT lines.

\section{Organ-specific and hormone-induced expression} of $\mathrm{ZmLOX12}$ and $\mathrm{ZmLOX12-dependent} \mathrm{oxylipin} \mathrm{signature}$ in mesocotyls.

To guide functional analyses of $Z m L O X 12$, we measured accumulation of its transcript in various maize organs and tissues, using massively parallel signature sequencing (MPSS) technology. The MPSS technique uses a 17-base tag signature to discriminate against highly homologous sequences, ensuring accurate detection of mRNA accumulation. ZmLOX12 transcripts were present at similar concentrations (approximately 25 parts per million [ppm]) in below-ground, vegetative, repro- ductive, and kernel tissues (Fig. 4A; measured as mRNA in ppm). While ZmLOX12 transcript abundance was relatively similar for many tissues tested (approximately $25 \mathrm{ppm}$ ), markedly high levels were detected in mesocotyls (200 ppm), indicating a potentially strong function for ZmLOX12 in that organ. To investigate the impact of ZmLOX12 on oxylipin production in mesocotyls, we profiled 9- and 13-oxylipins in WT and lox 12 mutants. Importantly, the oxylipin molecular species were quantified only for those molecules, for which commercial standards were available. This resulted in a large number of oxylipins that could not be measured. Despite the limited number of oxylipins measured, several molecules were found to accumulate differentially between the two genotypes. The 9-LOX-derived oxylipins 9-HODE, 9-HOTE [(9S,10E,12Z, 15Z)-9-hydroxy-10,12, 15-octadecatrienoic acid], and 9-KOTE were notably reduced in lox 12 mutant mesocotyls. Interestingly, 13-HODE [(9Z,11E,13S,15Z)-13-hydroxy-9,11,15ctadecadienoic acid], 13-KODE [keto (9Z,11E,13S,15Z)-13hydroxy-9,11,15-octadecadienoic acid], 13-HOTE [(9Z,11E, 13S, 15Z)-9-hydroxy-9,11,15-octadecatrienoic acid], and 13KOTE [keto (9Z,11E,13S,15Z)-9-hydroxy-9,11,15-octadecatrienoic acid] were also reduced in the mutants, indicating positive regulatory role for $\mathrm{ZmLOX} 12$-derived oxylipins in the biosynthesis of 13-oxylipins. Collectively, these results suggest

A

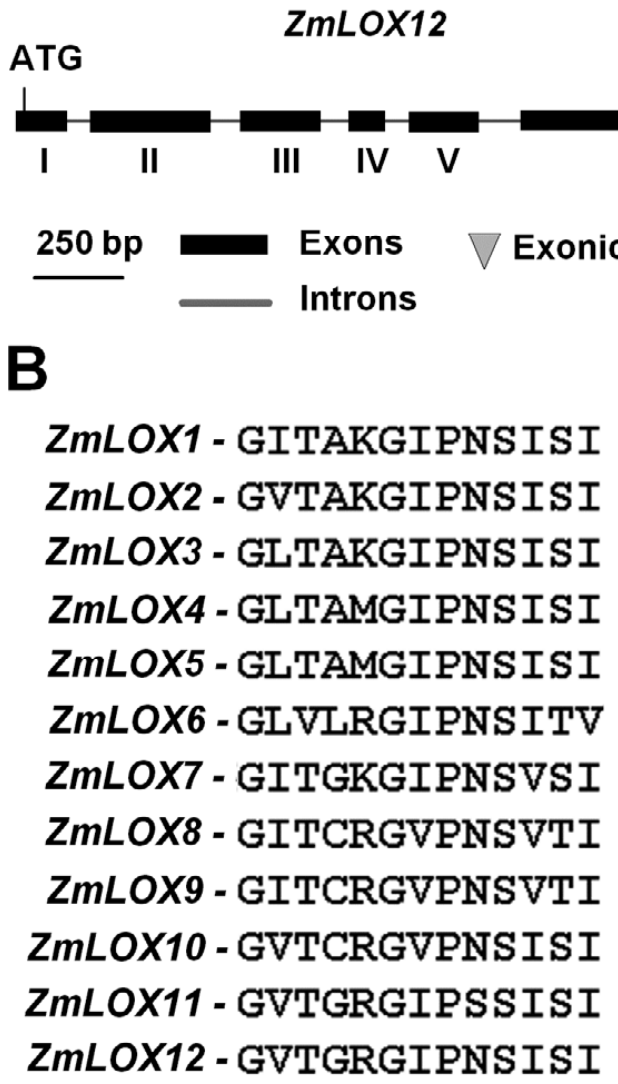

TGA VI $\underset{\text { Iox12-1 }}{\triangle}$

lox12-1
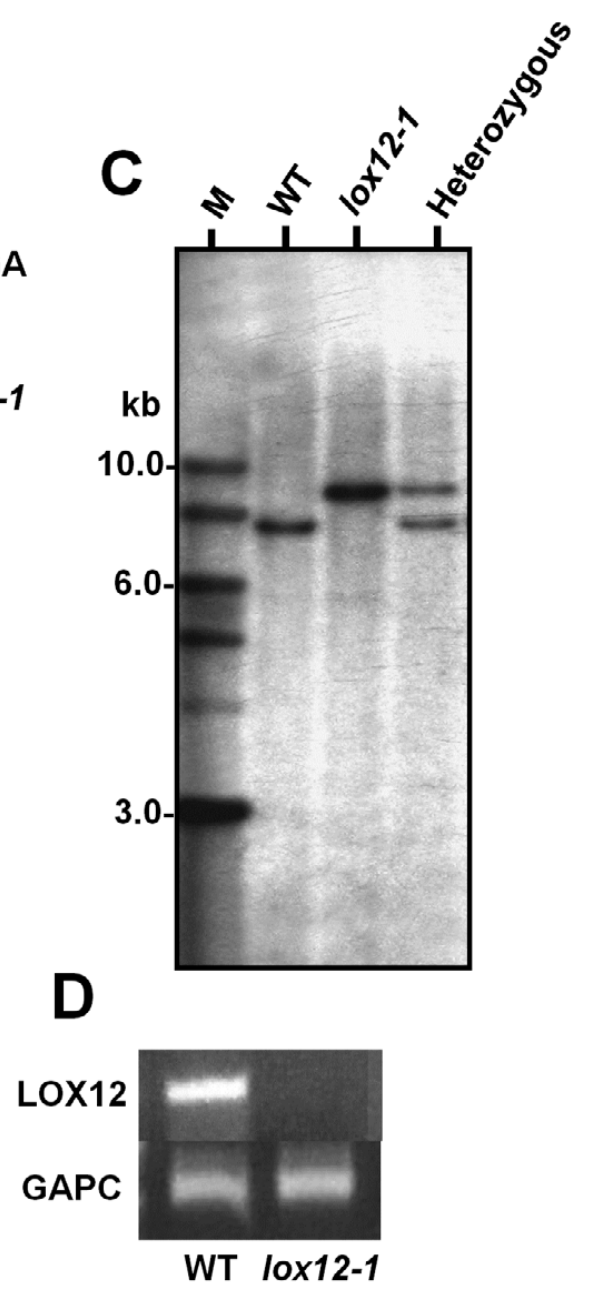

Fig. 3. Characterization of the lox12-1 mutant and C-terminus sequence alignment of the 12 maize lipoxygenases (LOX). A, Schematic representation of the genomic structure of $\mathrm{ZmLOX} 12$ showing the Mutator $(\mathrm{Mu}$ )-element insertion site. B, Amino acid sequence alignment of the $\mathrm{C}$-terminus for the 12 LOX in maize. C, Southern blot analysis of genomic DNA extracted from individual plants of a $\mathrm{BC}_{4} \mathrm{~F}_{2}$ family segregating for lox $12-1$ mutants and near-isogenic wild types (WT). Genomic DNA was digested with XbaI and was hybridized to the ZmLOX12 gene-specific probe. Lane order is as follows: molecular weight marker (M), wild type (WT); homozygous mutant (lox 12-1); and heterozygous. D, Semiquantitative reverse transcription-polymerase chain reaction analysis of ZmLOX12 transcript accumulation in mesocotyls of lox12-1 homozygous mutants and WT plants. No ZmLOX12-specific steady-state mRNA signal was detected in mesocotyls of lox 12-1 mutant seedlings, indicating that the lox12-1 is a true null allele of ZmLOX12. 
that $\mathrm{ZmLOX12}$ is a major isoform responsible for oxylipin biosynthesis in mesocotyls and that it is also expressed in other organs, including all of those targeted by $F$. verticillioides (roots, mesocotyls, stalks, and kernels).

9-LOX have been associated with biotic defense (Fammartino et al. 2007; Hwang and Hwang 2010; Lopez et al. 2011; Rance et al.1998; Vicente et al. 2012). Thus, we examined whether ZmLOX12 is transcriptionally activated in leaves by stressrelated hormones (JA, salicylic acid [SA], abscisic acid [ABA], and ethylene) and wounding. While the transcripts were barely detectable in untreated leaves, expression was
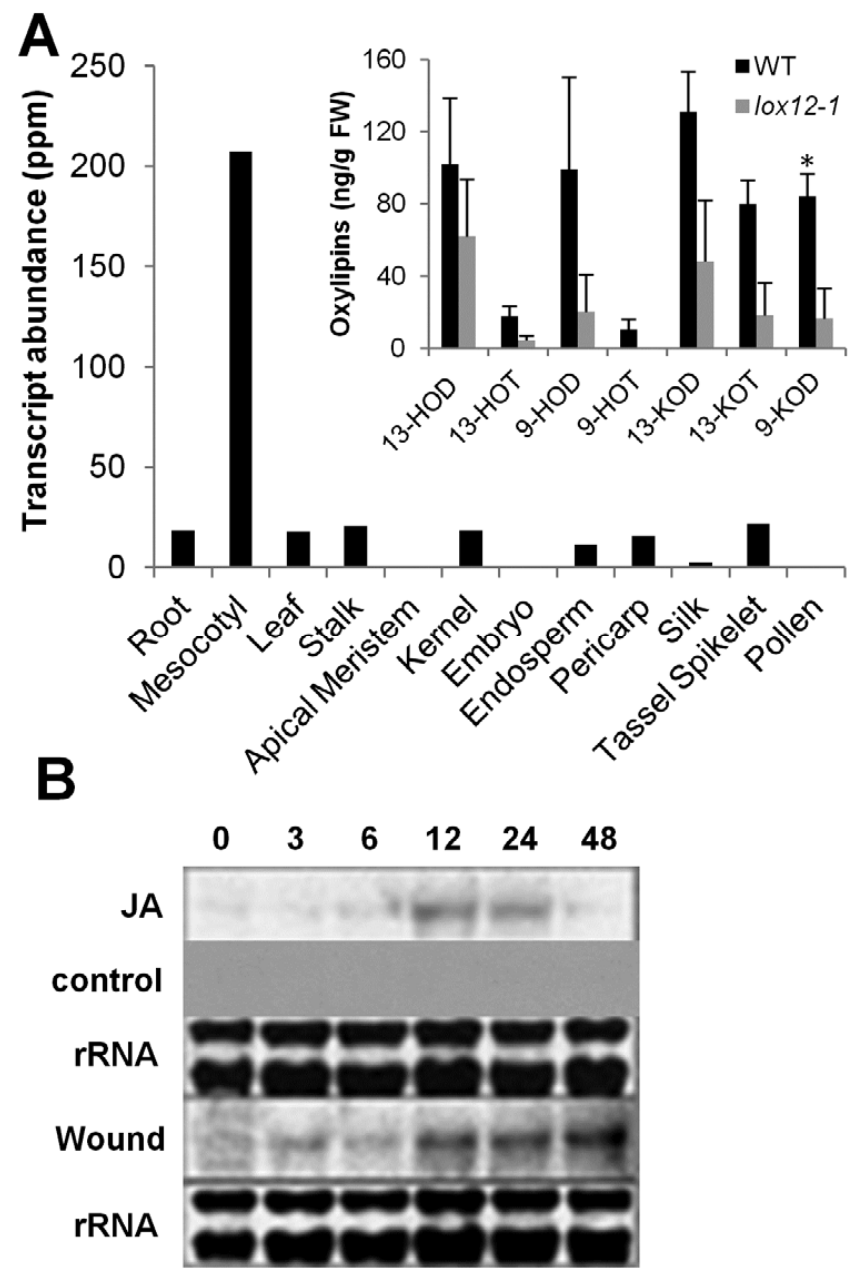

Fig. 4. Organ-specific and wound- and jasmonate-induced expression of ZmLOX12 and oxylipin profiling of lox12-1 mutants and wild-type (WT) mesocotyl. A, Massively parallel signature sequencing (MPSS) expression profile of $Z m L O X 12$ in different maize organs and oxylipin profiling in mesocotyls (Brenner et al., 2000). Expression of ZmLOX12 is plotted in parts per million (ppm) of transcript abundance in root, mesocotyl, leaf, stalk, apical meristem, kernel, embryo, endosperm, pericarp, silk, tassel spikelet, and pollen. Profiling of 9- and 13-oxylipins in WT and lox12 mutant mesocotyls. Oxylipin quantified included 9-HODE, (9S,10E,12Z)9-hydroxy-10,12-octadecadienoic acid; 9-KODE, ketodiene (9S,10E,12Z)9-hydroxy-10,12-octadecadienoic acid; 9-HOTE, (9S,10E,12Z,15Z)9-hydroxy-10,12, 15-octadecatrienoic acid; 13-HODE, (9Z,11E,13S, 15Z)-13-hydroxy-9,11,15-ctadecadienoic acid; 13-KODE, keto (9Z,11E, 13S, 15Z)-13-hydroxy-9,11,15-octadecadienoic acid; 13-HOTE, (9Z,11E, 13S, 15Z)-9-hydroxy-9,11,15-octadecatrienoic acid; and 13-KOTE, keto (9Z,11E,13S,15Z)-9-hydroxy-9,11,15-octadecatrienoic acid. B, Northern blot analysis of transcript levels of $Z m L O X 12$ in leaves in response to 200 $\mu \mathrm{M}$ jasmonic acid (JA) or wounding treatments. Tissues were harvested from leaves of seedlings at the V2 developmental stage. Blots were hybridized to the ${ }^{32} \mathrm{P}$-labeled $\mathrm{ZmLOX} 12$ probe. Equal loading of RNA was visually confirmed by ethidium bromide staining of ribosomal RNA (rRNA). induced to increased levels in response to wounding and treatment with JA (Fig. 4B) but not with SA, ABA, or ethylene. Mechanical wounding caused an earlier induction of $\mathrm{ZmLOX12}$ than JA treatment, suggesting that, in addition to JA, woundinduced $\mathrm{ZmLOX} 12$ expression may be regulated by other signals.

\section{Mesocotyls of lox12-1 mutants are susceptible to $F$. verticillioides.}

As an underground organ, mesocotyls are often subject to infection by seed- and soil-borne pathogens. Induction of ZmLOX12 transcripts was most strongly observed at 3 and $6 \mathrm{~h}$ following $F$. verticillioides inoculation, suggesting a role for this gene in maize defense (Fig. 5A). To determine the function of ZmLOX12 in defense against $F$. verticillioides, mesocotyls of lox12-1 mutant and WT seedlings were inoculated with fungal conidia. Disease development in infected and mock-treated plants was monitored for 8 days. Large rotted areas were clearly visible on lox12-1 mutant mesocotyls 6 and 8 days postinoculation (Fig. 5B and $\mathrm{C}$ ). In addition to the rotting observed in mesocotyls, blighting symptoms also developed in most mutant leaves (Fig. 5C). Near-isogenic WT seedlings exhibited only minor disease symptoms (Fig. 5B and C). Mock-inoculated mesocotyls were free of visible fungal colonization. These data provide evidence that $Z m L O X 12$ is necessary for defense against $F$. verticillioides in maize underground organs at the early stages of plant development.

To determine the biochemical mechanisms behind ZmLOX12-mediated resistance to $F$. verticillioides, we screened mutant and WT plants for differences in a range of oxylipins and plant hormones including JA, ABA, auxin, and SA. Among the hormones measured, major differences were observed for the cyclopentenone precursor of JA (12-OPDA) and the bioactive form of jasmonates (JA-Ile) with four- and sixfold lower levels found in lox 12-1 mutants $32 \mathrm{~h}$ postinfection, respectively (Fig. 6A). Transcript analysis showed that ZmLOX10, a 13-LOX recently reported to regulate woundinduced JA biosynthesis (Christensen et al. 2013), was induced 30 -fold above controls by $F$. verticillioides treatment in WT mesocotyls (Fig. 6B). Conversely, lox12-1 mutants exhibited ZmLOX10 expression levels similar to mock controls. An OPDA reductase (OPR) involved in JA biosynthesis, ZmOPR8 (Yan et al. 2012), also exhibited reduced transcript levels in lox12-1 mutants in response to infection. Collectively, these results suggest a positive role for ZmLOX12 regulation of pathogen-induced JA biosynthesis in response to $F$. verticillioides infection in mesocotyls and support previously shown defensive roles for 12-OPDA and JA-Ile against Fusarium species (Stintzi et al. 2001; Zhou et al. 2011). Surprisingly, no difference in 9-oxylipin content was observed between mesocotyls of WT and lox12-1 mutants during infection (Supplementary Fig. S4), suggesting that some other 9-oxylipins (not measured due to lack of standards) are the ZmLOX12 products induced in response to $F$. verticillioides infection.

\section{lox12-1 mutants accumulate increased kauralexins upon infection with $F$. verticillioides.}

As part of a more recent discovery in maize-biotic interactions, acidic diterpenoid phytoalexins, termed kauralexins, have been shown to accumulate to high levels in diseased tissues following fungal infection and stem boring by insects (Schmelz et al. 2011). We have monitored kauralexins A1-A3 and B1-B3 levels at 0,2, 4, and 6 days after infection of WT and lox12-1 mesocotyls with $F$. verticillioides. As expected, there was a gradual increase in kauralexin levels during disease progression (Fig. 7). In general, total levels of kauralexins were higher in F. verticillioides-infected lox12-1 tissues than 
WT at all timepoints (Supplementary Fig. S3). By 2 days posttreatment, infected lox12-1 mutants had significantly higher levels of kauralexin A1 (KA1) and KB1 than infected WT and noninfected mocks $(P<0.05)$ (Fig. 7). At 4 days postinoculation, the lox12-1 mutant contained significantly greater kauralexin levels than infected and control WT or mock-treated mutant $(P<0.05)$, whereas WT infected was not significantly different. By 6 days postinfection, kauralexin levels in the lox12-1 mutant were notably higher than WT infected tissues, with significant differences seen in levels of KA2 and KA3 $(P<$ $0.05)$. As kauralexins accumulate to higher levels in more diseased-tissues (Schmelz et al. 2011), our results suggest that accumulation of kauralexins may serve as an indicator of disease progression.

\section{lox 12-1 mutants are susceptible}

to $\boldsymbol{F}$. verticillioides infection of kernels and stalks.

Previous studies have shown that $F$. verticillioides causes disease in multiple maize organs, including kernels and stalks (Gao et al. 2007; Munkvold et al. 1997; Oren et al. 2003). With a clear function in mesocotyl defense, we hypothesized that $Z m L O X 12$ contributes to resistance against $F$. verticillioides in other organs. To test our hypothesis, we first carried out $F$. verticillioides kernel infection bioassays and measured conidiation and fumonisin $\mathrm{B}_{1}\left(\mathrm{FB}_{1}\right)$ production as described in Christensen and associates (2012). At 9 days postinoculation, lox12-1 mutant kernels had significantly higher levels of vegetative growth (Fig. 8A), sporulation (Fig. 8B), and $\mathrm{FB}_{1}$ production (Fig. 8C) than WT, indicating that ZmLOX12 mediates inhibition of fungal growth and mycotoxin production. Analysis of stalk infection showed similar results in that lox12-1 mutant plants were much more colonized by $F$. verticillioides than WT, with lesions being larger than $100 \mathrm{~mm}^{2}$ in more than $55 \%$ of the mutant stalks, compared with only $20 \%$ larger than $100 \mathrm{~mm}^{2}$ in WT stalks (Fig. 8D). Collectively, these results demonstrate that ZmLOX12 is a major defense gene against $F$. verticillioides colonization of maize kernels and stalks.

\section{JA-deficient opr7 opr8 double mutant lost immunity to $F$. verticillioides in mesocotyls.}

Because JA has not been previously reported to be an active regulator of plant defense against $F$. verticillioides, we investigated the function of JA by utilizing the recently published opr 7 opr 8 double mutant, which is JA-deficient in all maize organs, regardless of the stress imposed (Yan et al. 2012). In a F. verticillioides mesocotyl infection assay, strong rotting symptoms accompanied by visual mycelial growth was observed on opr 7 opr 8 mutants as early as 1 day postinfection, whereas WT mesocotyls showed no evidence of disease even at 2 days postinfection (Fig. 9A). Quantification of fungal biomass by ergosterol confirmed that opr 7 opr 8 double mutants were heavily colonized by $F$. verticillioides 2 days postinfection, while WT ergosterol levels were below the detectable limit. These results suggest that JA deficiency renders maize mesocotyls completely susceptible to $F$. verticillioides, which, at least in part, conceptually parallels our previous report showing that $o p r 7$ opr 8 mutants are unable to survive in nature due to the complete lack of immunity to oomycete Pythium spp. (Yan et al. 2012)

\section{DISCUSSION}

Despite the progress in understanding the role of specific 9LOX in dicots, the function of these enzymes in monocot spe-


Fig. 5. The Fusarium verticillioides-inducible gene $\mathrm{ZmLOX} 12$ is required for resistance in maize mesocotyls. A, Northern blot analysis of ZmLOX12 transcript accumulation in response to $F$. verticillioides infection, shown in hours postinfection (hpi). To obtain elongated mesocotyls, plants were germinated and grown in the dark for 10 days. Total RNA was extracted from mesocotyls after infection with $F$. verticillioides (three inoculation sites per mesocotyl with $10 \mu \mathrm{l}$ of $5 \times 10^{6}$ microconidia/ml per site) at indicated timepoints in hours. B, and $\mathbf{C}$, Disruption of the $\mathrm{ZmLOX} 12$ gene results in enhanced decay of mesocotyls caused by $F$. verticillioides infection, as compared to wild-type (WT) mesocotyls. Photos were taken 6 (B) and 8 (C) days postinoculation. Experiment was repeated two times, each containing at least 10 biological replicates. 
cies remains largely unexplored. Here, we report on the cloning and analysis of the molecular and biochemical properties of a novel maize 9-LOX, ZmLOX12, and provide genetic evidence for a role of this particular 9-LOX in maize resistance to the colonization of mesocotyls, kernels, and stalks by the soiland seed-borne pathogen $F$. verticillioides.

The absence of a recognizable chloroplast signal peptide and a relatively higher overall sequence identity to other 9LOX, as compared with 13-LOX, suggested that the ZmLOX12 predicted protein is a 9-LOX. This assumption was supported further by the analysis of regiospecificity of the recombinant ZmLOX12 protein, which clearly indicates that this protein is a linoleate 9-LOX. Low sequence homology of ZmLOX12 to other maize 9-LOX suggests its uniqueness (Supplementary Fig. S2). This observation was supported by sequence analysis that revealed the presence of the T/M motif instead of the conventional $\mathrm{T} / \mathrm{V}$ known to be indicative of 9LOX regiospecificity of all previously described 9-LOX (Feussner and Wasternack 2002). Interestingly, Southern blot analysis demonstrated that genes homologous to $\mathrm{ZmLOX12}$ were also present in other monocot species, thus suggesting that $Z m L O X 12$ homologs are distributed across many monocot species. The uniqueness of $Z m L O X 12$ is further accentuated by its strong linkage disequilibrium, which is extended further than what is common in maize (De la Fuente et al. 2013), indicating that this gene has been highly selected for by breeders since domestication.

The agronomic significance of controlling $F$. verticillioides infection and fumonisin contamination makes resistance to this pathogen an important selection trait. Root, mesocotyl, crown, and stalk were proposed to be the major routes for systemic infection of maize by $F$. verticillioides (Munkvold et al. 1997). In this study, we showed that ZmLOX12 transcripts were upregulated by infection of mesocotyls with $F$. verticillioides. This suggests that ZmLOX12 may be involved in maize defense mechanisms against this fungus. This was further strengthened by the fact that disruption of this gene resulted in a breakdown of resistance to $F$. verticillioides infection, as manifested by the enhanced decay of mesocotyls and, eventually, the entire seedlings. This finding provides evidence that $\mathrm{ZmLOX} 12$ has a


Fig. 6. lox12-1 mutants have reduced levels of jasmonates and jasmonate-biosynthetic gene expression. A, Liquid chromatography-tandem mass spectrometry analysis of 12-oxo phytodienoic acid (OPDA), jasmonic acid (JA), and JA-isoleucine (JA-Ile) levels in mock and Fusarium verticillioides-inoculated wild-type (WT) and lox12-1 mutant mesocotyls. B, Quantitative polymerase chain reaction analysis of jasmonate-biosynthetic genes ZmLOX10 and ZmOPR8 in mock and F. verticillioides-inoculated WT and lox12-1 mutant mesocotyls. Tissues were collected at 0, 32, and $96 \mathrm{~h}$ postinoculation. Bars are means \pm standard error of the mean; $n=4$ biological replicates with two plants per replicate; Tukey pairwise comparison; letters indicate significant differences, $P<0.05)$ 
significant role in the resistance of maize underground organs to $F$. verticillioides infection. Given that $F$. verticillioides is a common soil-borne pathogen found throughout the world, perhaps one reason $Z m L O X 12$ was strongly selected for during domestication (De La Fuente et al. 2013) was its function in preventing severe Fusarium rotting in maize.

The most likely cause of decreased resistance of lox $12-1$ mutants to $F$. verticillioides is substantially reduced levels of the pathogen-induced jasmonates OPDA and JA-Ile (Fig. 6). Reduced expression of the JA-regulating gene ZmLOX10 and the JA biosynthesis gene $Z m O P R 8$ in lox 12-1 mutants provides a possible mechanism for the reduced jasmonate production. The conclusive evidence demonstrating the central role of JA in resistance to $F$. verticillioides was obtained by analyses of JA-deficient opr 7 opr 8 double mutants, which are unable to produce detectable levels of JA in any maize organ tested (Yan et al. 2012). Remarkably, unlike the slow rotting symptoms observed in lox 12-1 mutants, which lasted up to 8 days, opr7 op8 double mutants displayed lack of immunity to this pathogen as evidenced by complete rotting of the mesocotyls within
2 days after infection (Fig. 9). Collectively, our results provide strong genetic evidence that the major defense hormone against $F$. verticillioides is JA. While to date, $F$. verticillioides has not conclusively been assigned any specific lifestyle, the requirement of JA for defense suggests that $F$. verticillioides is likely either a hemibiotroph or a necrotroph.

The ability of 9-LOX enzymes to influence 13-LOX oxylipin production was shown for another root-expressed 9-LOX of maize, ZmLOX3. The knockout lox3-4 mutant displayed higher levels of JA in the roots (Gao et al. 2008) and increased resistance to a root-rotting pathogen Exherohilum pedicelatum (Isakeit et al. 2007). Unlike the reported negative role of ZmLOX3 in the regulation of JA in roots, the current study demonstrates that another 9-LOX pathway branch initiated by ZmLOX12 is required for positive regulation of jasmonate production.

The requirement of JA-mediated defense against $F$. verticillioides suggests a curious distinction between the two most economically important Fusarium species. Two previous reports, using mutants in the AtCOI1 and AtPFT1 genes,


Fig. 7. lox12-1 mutants have increased levels of kauralexins upon infection with Fusarium verticillioides. Average $(n=4)$ induced kauralexin A1-A3 and B1B3 concentrations in wild-type (WT) and lox12-1 mutant mesocotyls with mock-inoculated (WT, white; lox12-1, dark gray), and F. verticillioides-infected (WT, light gray; lox12-1, black) samples at $0,2,4$, and 6 days postinoculation. Pairwise comparisons were made within groups at each timepoint (mean \pm standard error of the mean, Tukey pairwise comparison). Letters indicate significant differences $(P<0.05)$. 
showed that Arabidopsis plants compromised in JA defense signaling are more resistant to the root-infecting fungal pathogen Fusarium oxysporum (Kidd et al. 2009; Thatcher et al. 2009), indicating that $F$. oxysporum pathogenicity is largely jasmonate-dependent. Similarly, mutants of AtCOI1, AtJAR1, and AtOPR3 were more resistant to $F$. graminearum (Makandar et al. 2010). In this study, we demonstrate that the ability of $F$. verticillioides to infect maize is largely subdued by an intact JA-mediated defense system. Comparatively, these studies demonstrate the diverse nature by which plant pathogens (even from the same genus) can specialize pathogenicity strategies so that the same host hormone signal may either serve to provide major defense or can be taken advantage of to facilitate pathogenesis.

Interestingly, WT plants produced substantially higher jasmonate levels yet yielded lower levels of kauralexins, which reaffirms that jasmonates alone are not sufficient to activate kauralexin biosynthesis, as previously reported (Schmelz et al. 2011). As kauralexins have been shown to be defensive phytoalexins (Schmelz et al. 2011), it is curious that lox12-1 mutants had significantly increased levels of these compounds, yet, were more susceptible. This is likely due to the ability of $F$. verticillioides to detoxify kauralexins in order to advance infectious processes. For example, Fusarium sambucinum is known to effectively detoxify terpenoid phytoalexins to facilitate dry rot in potato (Desjardins et al. 1992). These examples demonstrate that defensive phytoalexins might not always sufficiently thwart pathogenicity, but rather, other defense measures such as oxylipin-derived defenses or signals may be required to confer resistance.

Because contamination of maize kernels with fungal mycotoxins is a significant agroeconomic problem, it was important to test whether disruption of this gene had any effect on fumonisin production in kernels infected by $F$. verticillioides. Kernel bioassays showed that fumonisin $B_{1}$ levels were increased almost 20-fold in the lox12-1 mutant kernels compared with near-isogenic WT kernels. A similar pattern was also seen in stalk rot assays in which lox 12 mutants were more susceptible as compared with WT plants. To the best of our knowledge, no previous genetic study has shown the significance of a single maize gene in defense against $F$. verticillioides. Although the present study focused on the role of ZmLOX12 in maize- $F$. verticillioides interactions in the stalk, mesocotyls, and seed, the role of this gene may not be solely restricted to these organs or this particular pathogen. For example, we showed transient induction of ZmLOX12 mRNA accumulation in leaves by wounding and exogenous application of JA, suggesting the possibility of a defense-related function in leaves in response to pests or necro- or hemibiotrophic pathogens. Although the precise oxylipin molecules by which ZmLOX12 contributes to enhanced JA and subsequent maize resistance remains to be elucidated, our discovery of a key defense gene against $F$. verticillioides will help advance novel candidate gene-based strategies to develop superior maize lines for controlling maize Fusarium stalk and ear rots and fumonisin contamination in kernels caused by $F$. verticillioides.

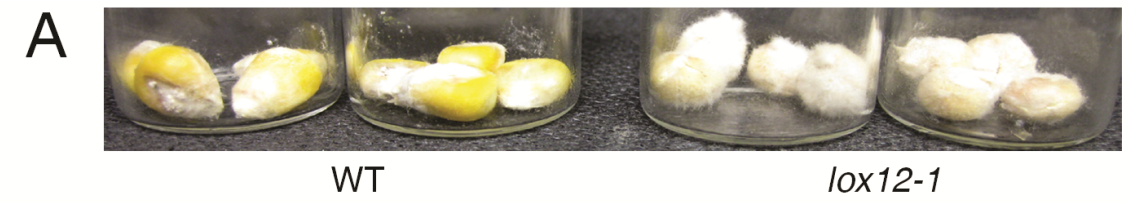

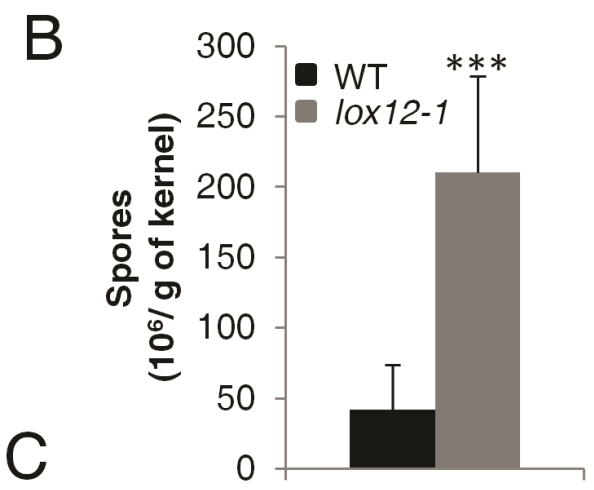

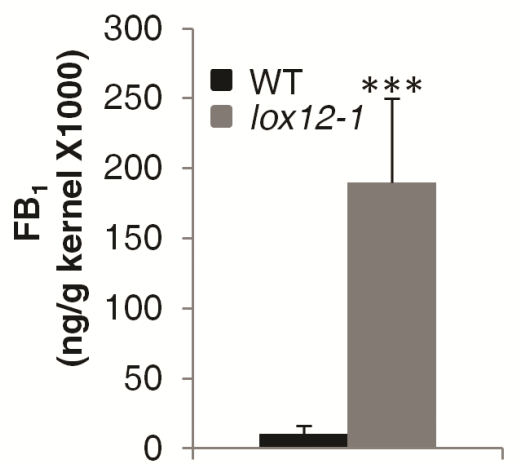

Fig. 8. lox12-1 mutant is susceptible to Fusarium verticillioides colonization, conidiation, and fumonisin $\mathrm{B}_{1}\left(\mathrm{FB}_{1}\right)$ production. $\mathbf{A}$, Mycelial growth of $F$. verticillioides on wild-type (WT) and lox12-1 mutant kernels. $\mathbf{B}$ and $\mathbf{C}$, Quantification of conidiation and $\mathrm{FB}_{1}(n=5$; analysis of variance, *** $P \leq 0.001)$. All experiments were carried out to 9 days postinoculation. $\mathrm{FB}_{1}$ values are $\times 1,000$. D, Disease symptoms on WT and lox $12-1$ mutant stalks infected with $F$. verticillioides $(n=5)$. Photos were taken 9 days postinfection and lesions were quantified using ImageJ software. Following quantification, internodes were placed into two separate categories, $<100 \mathrm{~mm}^{2}$ and $>100 \mathrm{~mm}^{2}$. 


\section{MATERIALS AND METHODS}

Plant material and treatments.

Maize plants were grown in 7-cm pots in Strong-Lite potting soil (Universal Mix, Pine Bluff, AK, U.S.A.) at 22 to $30^{\circ} \mathrm{C}$ under a 16-h-day length, 50\% average relative humidity, and 560 to $620 \mu \mathrm{E}$ of light. For all organ-specific expression studies, wounding, and hormonal treatments, 2-week-old maize (Zea mays L.) seedlings of the inbred line B73 at the V2 developmental stage were used. For wounding experiments, the second fully expanded leaves of B73 seedlings at V3 stage were wounded by crushing the lamina with a hemostat. For hormonal treatments, the seedlings were cut at the soil level and were incubated with the cut end placed in $100 \mathrm{ml}$ of either $200 \mu \mathrm{M} \mathrm{JA}, 2.5 \mathrm{mM} \mathrm{SA}$, $100 \mu \mathrm{M}$ ABA, or $0.01 \%$ Tween-20 as a control (Sigma, St. Louis). Treatment with $10 \mu$ of ethylene per liter was conducted in hermetically sealed 5.6-liter desiccators.

\section{Isolation of genomic DNA and Southern blot analysis.}

Freshly harvested leaves were used for extraction of genomic DNA as described by Zhang and associates (2005).
For Southern blot assay, genomic DNA $(10 \mu \mathrm{g})$ from the plant species listed above was cut with the restriction enzyme XbaI. Digested DNA was separated on a $0.8 \%$ agarose electrophoresis gel, was transferred $(0.025 \mathrm{M}$ phosphate transfer buffer) and, then, cross-linked to the nylon membrane (Magna nylon transfer membrane; Osmonics Inc., Minnetonka, MN, U.S.A.) by UV Stratalinker 2400. The blots were hybridized overnight at $42^{\circ} \mathrm{C}$ with a ${ }^{32} \mathrm{P}$-labeled $\mathrm{ZmLOX} 12$ specific probe. Original EST p0006.camah52 2,689-bp long clone containing almost the full-length cDNA sequence, lacking only $24 \mathrm{bp}$ after the start codon, was used as a template for labeling the probe. The membrane was hybridized in ULTRAhyb hybridization buffer (Ambion, Austin, TX, U.S.A.). Washes were performed; first with low-stringency buffer $(2 \times$ SSC [ $1 \times \mathrm{SSC}$ is $0.15 \mathrm{M} \mathrm{NaCl}$ plus $0.015 \mathrm{M}$ sodium citrate] and $0.1 \%$ sodium dodecyl sulfate [SDS]) at $42^{\circ} \mathrm{C}$, twice for 5 min, followed by an additional wash with high-stringency buffer $(0.2 \mathrm{M} \mathrm{SSC}$ and $0.1 \% \mathrm{SDS})$ at $42^{\circ} \mathrm{C}$, twice for $15 \mathrm{~min}$. The blots were exposed to X-ray film (Kodak, Rochester, NY, U.S.A.) in cassettes at $-80^{\circ} \mathrm{C}$ for $72 \mathrm{~h}$, unless otherwise indicated.
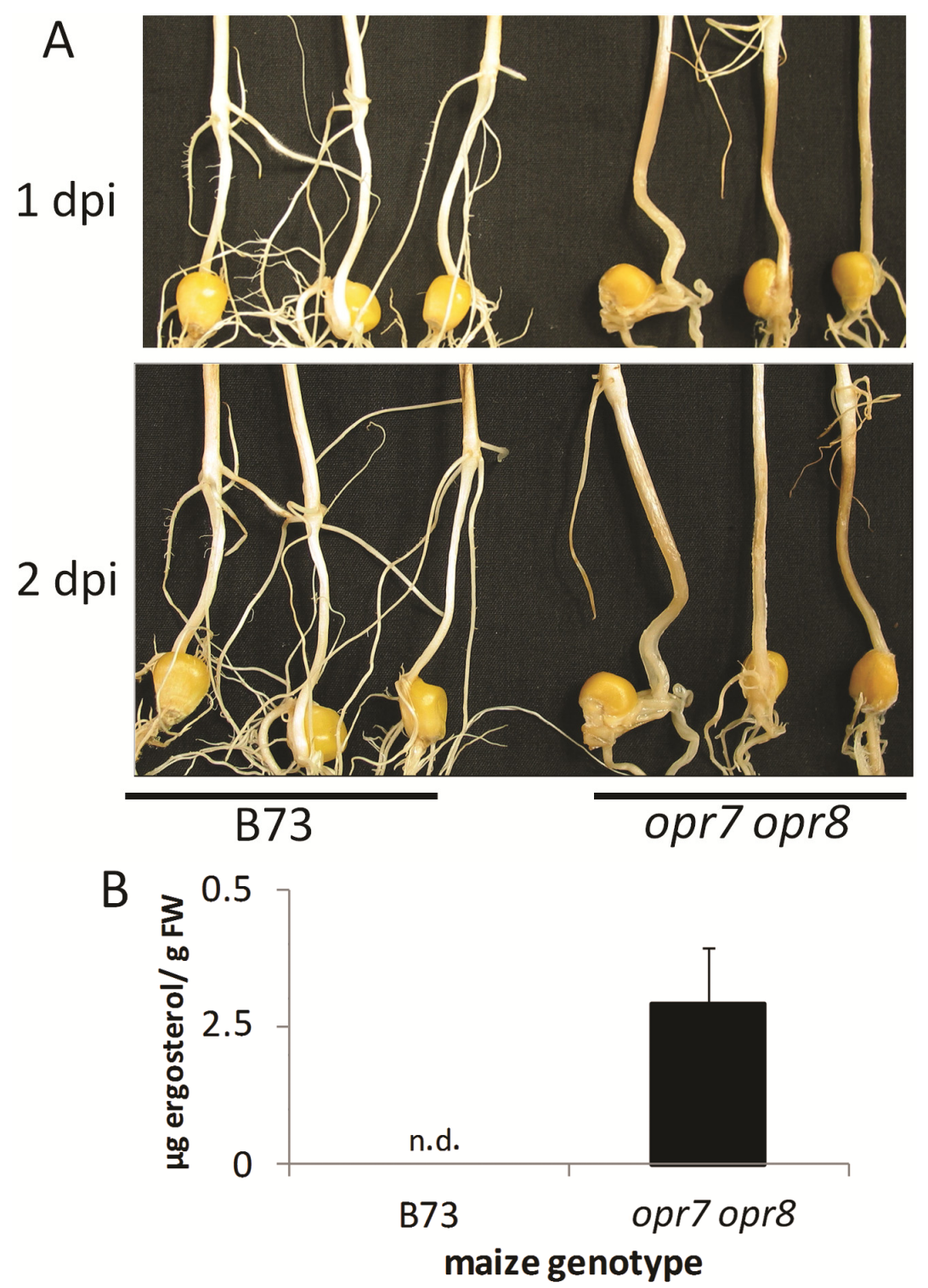

Fig. 9. Fusarium verticillioides colonization of mesocotyls of wild type (WT) and jasmonic acid (JA)-deficient, opr7 opr8 double mutant. A, Visual comparison of WT and opr7 opr8 double mutant mesocotyls inoculated with F. verticillioides at 1 and 2 days postinoculation. B, Fungal colonization of mesocotyls as measured by quantification of the fungus-specific membrane lipid ergosterol. 
Bioinformatics analysis.

A homology search using ZmLOX12-translated sequences was done using the BLAST search that is publicly available at the National Center for Biotechnology Information (NCBI) (Bethesda, MD, U.S.A.). Percent identity of ZmLOX12 with other plant LOX was evaluated using ClustalW 1.8 software (Chenna et al. 2003).

Subcellular localization was predicted based on the identification of signal peptide sequences by two different programs, ProtComp 6.0 (Softberry, Inc. Mount Kisco, NY, U.S.A.) and Proteome Analyst-Subcellular Specialization server 2.5 (Lu et al. 2004). Presence of conserved domains in the deduced amino acid sequences was determined by a NCBI-powered search using the Conserved Domain Database (MarchlerBauer et al. 2005) and Pfam 20.0 software (Finn et al. 2006). To determine the genomic structure of $\mathrm{ZmLOX12}$, a homologybased BLAST search was performed by utilizing the databases of the maize Genome Survey Sequences database available at NCBI, the Plant Genome database (Dong et al. 2004), The TIGR Maize database (Sutton et al. 1995), as well as Maize Assembled Genomic Island databases (Emrich et al. 2004).

\section{Construction of the overexpression vector and analysis of ZmLOX12 biochemical properties.}

The entire open reading frame was PCR-amplified from root-specific cDNA using primers with adapted in-frame EcoRI-sites 5'-CTT-TGA-ATT-CAT-GCA-AAT-GCC-CTTCTG-TC- $3^{\prime}$ and 5'-CGA-GAA-TTC-CAA-TTG-TCA-TATGGA-GAC-GCT-G-3'. Following separation and elution from gel, the purified fragment harboring the $\mathrm{ZmLOX} 12$ coding sequence was ligated into the pCR2.1 TOPO vector (Invitrogen, Carlsbad, CA, U.S.A.). After restriction digestion with EcoRI, the coding region of $Z m L O X 12$ was placed under the control of the IPTG-inducible promoter of $E$. coli expression vector pET28a containing ampicillin-positive and kanamycin-positive resistance genes as selection markers (EMD Biosciences, Inc., San Diego, CA, U.S.A.) using T4 DNA ligase (Roche Applied Science, Indianapolis, IN, U.S.A.). After ligation, the ZmLOX12/ pET28a expression construct was heat-shock transformed into the BL21 (DE3) strain of E. coli chemically competent cells (EMD Biosciences, Inc.). Two independent E. coli BL21 (DE3) cultures containing the expression constructs were grown overnight at $37^{\circ} \mathrm{C}$ in $20 \mathrm{ml}$ of Luria Bertani medium containing $50 \mu \mathrm{g}$ of ampicillin per milliliter and $25 \mu \mathrm{g}$ of kanamycin per milliliter. When the cell culture reached the absorption value at $600 \mathrm{~nm}=0.7,1 \mathrm{mM}$ IPTG was added to induce expression of the constructs at $15^{\circ} \mathrm{C}$ for $48 \mathrm{~h}$. Cells collected from $200 \mathrm{ml}$ of culture were resuspended in $30 \mathrm{ml}$ of lysis buffer $(50 \mathrm{mM}$ Tris-HCL, $\mathrm{pH} \mathrm{7.5)}$ that contained $10 \%$ (vol/vol) glycerol, $0.5 \mathrm{M} \mathrm{NaCl}$, and $0.1 \%$ Tween-20. Cells were then disrupted using a sonifier tip with a frequency of 5 pulses per $30 \mathrm{~s}$. To remove cellular debris, samples were centrifuged at $12,000 \times g$ for $15 \mathrm{~min}$. The supernatant was divided into $1-\mathrm{ml}$ aliquots and were stored at $-20^{\circ} \mathrm{C}$ (Feussner et al. 1998). Oxygenation of linoleic acid was carried out by incubating the crude extract of bacteria containing the $Z m L O X 12$ overexpression construct with the substrate (120 $\mu \mathrm{M}$ final concentration) diluted in $1 \mathrm{ml}$ of $0.1 \mathrm{M}$ sodium phosphate buffer ( $\mathrm{pH} 5.7$ to 7.0 ) or in $0.1 \mathrm{M}$ Tris buffer ( $\mathrm{pH} 7.7$ to 8.6 ) for $20 \mathrm{~min}$ at room temperature. The reaction was stopped by the addition of $100 \mu \mathrm{l}$ of glacial acid. After centrifugation, the organic phase was removed under vacuum and remaining lipids were resuspended in $0.1 \mathrm{ml}$ of high-performance liquid chromatography (HPLC) solvent. Detection of LOX products by HPLC was carried out on an Agilent (Waldbronn, Germany) 1100 HPLC system, on which absorbency was recorded simultaneously at 234 and $210 \mathrm{~nm}$. The enantiomer composition of the hydroxyl fatty acids was analyzed as described by Feussner and Kühn (1995).

\section{Fusarium verticillioides infection of mesocotyls.}

Two-week-old seedlings grown in the dark for etiolated mesocotyls were used to study the gene expression of ZmLOX12 during $F$. verticillioides infection. For all studies related to $F$. verticillioides infection, we used strain 7600 (M3125; Fungal Genetics Stock Center, Kansas City, KS, U.S.A.), which was stored at $-80^{\circ} \mathrm{C}$ in $20 \%$ glycerin. For conidia production, the fungus was grown at $28^{\circ} \mathrm{C}$ on potato dextrose agar medium (B\&D, Sparks, MD, U.S.A.) as described by Shim and Woloshuk (1999). Suspensions containing $10^{6}$ conidia per milliliter in $0.1 \%$ Tween-20 were prepared by collecting conidia in $5 \mathrm{ml}$ of sterile water from 3-week-old V8 agar cultures and, then, filtering through four layers of miracloth. Control plants were inoculated with sterile water containing $0.1 \%$ Tween-20. Plant mesocotyls were first slightly wounded, using the syringe needle at three separate sites $(3 \mathrm{~cm}$ apart from each other) on each mesocotyl. $F$. verticillioides conidial suspension $(10 \mu \mathrm{l})$ was applied to each wound site on horizontally placed mesocotyls. Following inoculation, plants were immediately placed under a plastic cover to create a moist environment conducive to conidial germination. Inoculated and control plants were incubated for selected times under indicated conditions. After each incubation period, infected and control mesocotyls were collected and frozen immediately in liquid $\mathrm{N}_{2}$ and were stored at $-80^{\circ} \mathrm{C}$ until used for total RNA extractions.

\section{Kernel assays and stalk rot.}

Preparation of maize kernels, fungal inoculum, and inoculation procedure was done as described in Gao and associates (2007) and, most recently, in a methods paper by Christensen and associates (2012). For this analysis, we used $\mathrm{BC}_{3} \mathrm{~F}_{6}$ seeds of near-isogenic WT and a corresponding mutant in B73 genetic background. A suspension of $F$. verticillioides 7600 $\left(2.5 \times 10^{5}\right.$ conidia) was used for inoculation of each biological replicate. After nine days incubation at $28^{\circ} \mathrm{C}, \mathrm{FB}_{1}$ was extracted from kernels and was analyzed by HPLC as described in Shim and Woloshuk (2001). Two independent experiments were performed with five biological replicates, each containing four kernels and yielding similar results. Stalk rot assays were also performed as outlined by Gao and associates (2007). In brief, 10-week-old WT and lox12-1 mutant plants were wounded with a needle $(1 \mathrm{~cm}$ in depth) and were infected by placing a cotton swab soaked in $1 \times 10^{6} \mathrm{~F}$. verticillioides spores on the wound site and, subsequently, creating a humidity chamber by sealing it with Parafilm (Sigma, St. Louis). At 9 days postinoculation, 15 internodes per genotype were collected and lesion areas were measured using ImageJ software (ImageJ $1.36 \mathrm{~b}$; W. Rasband, NIH, Bethesda, MD, U.S.A.).

\section{RNA extraction, Northern blot analysis, and RNA profiling using MPSS, and EST.}

Total RNA from all maize tissues except kernels was extracted by using TRI reagent (Molecular Research Center Inc., Cincinnati, OH, U.S.A.), according to the protocol provided by the manufacturer. Kernel RNA was isolated using RNeasy plant mini kit (Qiagen, Valencia, CA, U.S.A.). After extraction, $10 \mu \mathrm{g}$ of total RNA from each genotype and treatment was separated using electrophoresis in $1.5 \%$ (wt/vol) formaldehyde agarose gels in $1 \times$ morpholinepropanesulfonic acid buffer and was then transferred onto a MagnaGraph nylon membrane (Micron Separations Inc., Westboro, MA, U.S.A.). To confirm equal loading of RNA samples into the agarose gel and uniform transfer onto the nylon membrane for all experiments, rRNA was stained with ethidium bromide and was 
visualized by UV light. Northern blot membranes were hybridized with ${ }^{32} \mathrm{P}$-labeled gene-specific probes using UltraHyb hybridization solution (Ambion) under conditions described in the manufacturer's protocol. Membranes were washed in $1 \times$ SSC, $0.1 \%$ SDS at room temperature for $15 \mathrm{~min}$, in $0.1 \times \mathrm{SSC}$, $0.1 \% \mathrm{SDS}$ at room temperature for $30 \mathrm{~min}$, followed by a final wash in $0.1 \times \mathrm{SSC}, 0.1 \% \mathrm{SDS}$ at $42^{\circ} \mathrm{C}$ for $30 \mathrm{~min}$. For autoradiography, RNA blots were exposed to a BioMax X-ray film (Kodak) for 2 to 7 days at $-80^{\circ} \mathrm{C}$. Blots presented in this manuscript are representative examples of at least two independent experiments. ZmLOX12 transcript expression levels were analyzed using MPSS technology from Lynx Technologies (Brenner et al. 2000). MPSS profiling entails transcript sampling (between 1 and 2 million sequenced transcripts per EST library) on microbeads, with a 17-base sequence read (tag) beginning with GATC. A total of 203 MPSS libraries covering diverse tissues, developmental stages, and treatments were assayed as described by Zhang and associates (2005).

\section{Generation of lox12 mutants.}

The reverse genetics resource (Trait Utility System for Corn [TUSC]) generated at DuPont Pioneer Hi-Bred International that represents a library of $M u$-transposon-mutagenized $\mathrm{F}_{2}$ was used to identify lox12 mutants (Meeley and Briggs 1995). Genomic DNA extracted from individual plants in this library was used to identify insertions in $\mathrm{ZmLOX} 12$ by PCR-based screening as described by Bomblies and associates (2003) with minor modifications. For this screening procedure, we used a combination of $M u$-terminal inverted repeat-specific primer (5'-AGA-GAA-GCC-AAC-GCC-AWC-GCC-TCY-A-3') with either of the ZmLOX12-specific primers: 61674 F5'-CCG-GCTCAT-GCG-CCT-GAG-AAC-3'; 61675 R 5'-CGT-TGG-ACTCTG-GCA-CCA-CGA-AG-3'; 61676 F 5'-TGT-CGT-TGCCGG-GGA-AGA-CTA-CCA-3'; 61677 F 5'-ACG-ATG-GCGTAC-CAG-GTG-ACC-TTC-3'; 61678 R 5'-TAG-TCT-ACTGAT-CAC-CAC-CGG-GTT-GAG-3'; 61679 F 5'-CTC-AACCCG-GTG-GTG-ATC-AGT-AGA-CT-3'; 61680 R 5'-GCTGAT-GAG-CTG-ATG-GTA-GCC-TGA-ATC-AT-3'; 61681 F 5'-CTT-GCT-AAG-GCT-CAT-GTC-TCC-GTC-AAT-GA-3'; 61682 R 5'-TCA-TAT-GGA-GAC-GCT-GTT-GGG-TAT-CCCT-3'; 61683 F 5'-ATG-GAG-ATG-TCC-TCG-GCC-ATC-TACTC-3'; 61684 R 5'-GAA-CAG-CAG-CGT-GTA-CGG-CACCTT-3'. Screening of the mutant library identified one PCRpositive individual. Fragments flanking both sides of the insertion were PCR-amplified, were cloned into the pCR2.1 TOPO vector (Invitrogen), and were sequenced to identify the location of the $M u$ insertion. Sequence analysis revealed that a $M u$ element was inserted 23 bp upstream of the stop codon. This mutant allele was designated lox $12-1$. In addition to the mutation in $\mathrm{ZmLOX12}$, it is highly probable that the lox 12 mutant individual contained a number of unrelated $M u$ elements elsewhere in the genome. Therefore, the original lox $12-1$ mutant allele was backcrossed three times $\left(\mathrm{BC}_{4}\right)$ to selected inbred lines to reduce the probability of these irrelevant mutations. Resultant $\mathrm{BC}_{4}$ individuals were self-pollinated to generate a $\mathrm{BC}_{4} \mathrm{~F}_{2}$ population of nearisogenic individuals in which homozygote mutants (lox121/lox12-1) and WT (LOX12/LOX12) as well as heterozygotes at the ZmLOX12 locus were identified by PCR and confirmed by Southern blot assays as described.

\section{Quantification of plant metabolites.}

Treated samples were prepared via extraction, methylation, vapor-phase extraction, and isobutene cylindrical inclusion gas chromatographic mass spectrometry-based mesocotyl quantification of defense metabolites (e.g., SA, JA, OPDA, and kauralexins) using methods described by Schmelz and associates (2003, 2011). Phytohormone extraction followed Christensen and associates (2013), with the following modifications: 500 $\mu \mathrm{l}$ of phytohormone extraction buffer (1-propanol/water/HCl [2:1:0.002 $\mathrm{vol} / \mathrm{vol} / \mathrm{v}])$ and $10 \mu \mathrm{l}$ of $5 \mu \mathrm{M}$ solution of deuterated internal standards: d-ABA $\left(\left[{ }^{2} \mathrm{H}_{6}\right](+)\right.$-cis,trans-ABA; [Olchem $\left.]\right)$, d-IAA $\left(\left[{ }^{2} \mathrm{H}_{5}\right]\right.$ indole-3- acetic acid, Olchem) and d-JA (2,4,4-d ${ }_{3}$; acetyl-2,2-d $\mathrm{d}_{2}$ JA; CDN Isotopes), and d-SA (d6-SA, Sigma) was added to approximately $100 \mathrm{mg}$ of ground mesocotyl tissue. Samples were agitated for $30 \mathrm{~min}$ at $4^{\circ} \mathrm{C}$ under darkness, and then, $500 \mu \mathrm{l}$ of dichloromethane was added to samples and they were agitated for $30 \mathrm{~min}$ at $4^{\circ} \mathrm{C}$ under darkness. Samples were centrifuged at $13,000 \times g$ for $5 \mathrm{~min}$ and the lower layer was collected into a glass vial for evaporation under a nitrogen gas stream. Samples were resuspended in $150 \mu \mathrm{l}$ of methanol and were centrifuged in a $1.5-\mathrm{ml}$ microcentrifuge tube at $14,000 \times g$ for 2 min to pellet any debris. Supernatant $(100 \mu \mathrm{l})$ was collected into an autosampler vial for direct injection into LCESI-MS/MS. The simultaneous detection of several hormones utilized methods by Muller and associates (2013) with modifications. The quantification utilized an Ascentis Express C-18 Column $(3 \mathrm{~cm} \times 2.1 \mathrm{~mm}, 2.7 \mu \mathrm{m})$ connected to an API 3200 LC-electrospray ionization-tandem mass spectrometry (MS/ MS) with multiple reaction mentoring (MRM). The injection volume was $2 \mu \mathrm{l}$ and had a $600 \mu \mathrm{l} / \mathrm{min}$ mobile phase consisting of Solution A $(0.05 \%$ acetic acid in water) and Solution B $(0.05 \%$ acetic acid in acetonitrile) with a gradient consisting of (time - \%B): $0.3-1 \%, 2-45 \%, 5-100 \%, 8-100 \%, 9-1 \%$, 11 - stop.

\section{Semiquantitative RT-PCR assay.}

Total RNA was extracted from mesocotyls of lox12-1 mutant and WT seedlings as described above. The RNA concentration was quantified using an ND-1000 spectrophotometer (Technologies Inc., Centerville, DE, U.S.A.). After removing contaminating DNA using DNA-free DNAse treatment kit (Ambion), RNA was equalized and $5 \mu \mathrm{g}$ was used for construction of cDNA using the First-Strand cDNA synthesis kit (GE Healthcare Bio-Sciences Corp., Piscataway, NJ, U.S.A.) following the manufacturer's protocol. A total of $500 \mathrm{ng}$ of each cDNA sample served as a template in a PCR assay using $\mathrm{ZmLOX12-}$ specific primers: 61683 5'-ATG-GAG-ATG-TCC-TCG-GCCATC-TAC-TC-3'; lox12utrR1 5'- CCA-AAC-CAA-TCA-TCGCAA-TAA-AAC-ATA-T-3'. The same conditions were used in semiquantitative RT-PCR analysis of $\mathrm{ZmLOX} 12$ expression in kernels. As a control for equal loading, primers specific to the housekeeping gene (GAPc; GeneBank accession number X07156) were used: GAPc5' 5'-GCT-AGC-TGC-ACC-ACAAAC-TGC-3' and GAPc3' 5'-TAG-CCC-CAC-TCG-TTGTCG-TAC-3'

\section{Quantitative real-time PCR analysis.}

Total RNA was extracted by RNeasy plant mini kit (Qiagen) according to manufacturer's specifications and was quantified by Nanodrop. Total RNA ( $5 \mu \mathrm{g})$ was DNAse treated (Thermo Scientific, Waltham, MA, U.S.A.) and was diluted to $4 \mathrm{ng} / \mu \mathrm{l}$ for quantitative PCR $(0.25 \mu \mathrm{l}$ of Verso enzyme mix, $12.5 \mu \mathrm{l}$ of $2 \times 1$-Step qPCR SYBR Mix, $1.25 \mu \mathrm{l}$ of RT Enhancer, $2 \mu \mathrm{l}$ of $1 \mu \mathrm{M}$ each forward and reverse primer, $1 \mu \mathrm{l}$ of $12.5 \mu \mathrm{M}$ ROX, $4.5 \mu \mathrm{l}$ of water, and $2 \mu \mathrm{l}$ of $4-\mathrm{ng} / \mu \mathrm{l}$ template; Thermo Scientific) as done by Constantino and associates (2013) and utilized Cullin as a reference gene.

\section{Ergosterol quantification.}

Ergosterol extractions were prepared by incubating infected mesocotyls with $1 \mathrm{ml}$ of $2: 1$ chloroform/methanol (vol/vol) for $24 \mathrm{~h}$ in darkness at room temperature. Extracts were filtered through a $0.2 \mu \mathrm{m}$ nylon membrane pore syringe filter, and 10 $\mu \mathrm{l}$ of filtrate were combined with $90 \mu \mathrm{l}$ of $5 \mu \mathrm{M} \mathrm{C}^{13}$-labeled 
cholesterol (cholesterol-25,26,27- ${ }^{13} \mathrm{C}$; Sigma) in methanol as internal standard. Ergosterol was quantified through LC/MS with an Ascentis Express C-18 column $(3 \mathrm{~cm} \times 2.1 \mathrm{~mm}, 2.7$ $\mu \mathrm{m})$ connected to an API $3200 \mathrm{LC} / \mathrm{MS} / \mathrm{MS}$ with atmospheric photochemical ionization and utilizing MRM (Headley et al. 2002). The injection volume was $2 \mu \mathrm{l}$ and the isocratic mobile phase consisted of methanol at a flow rate of $200 \mu \mathrm{l} / \mathrm{min}$.

\section{ACKNOWLEDGMENTS}

We thank M. Dickman, H. Alborn, and M. Shabab for critically reading the manuscript and for their valuable comments. Many thanks to $\mathrm{S}$. Murray and his group for their continuous and generous support of fieldbased genetic advancement of the maize mutants and W.-B. Shim for providing fungal cultures of $F$. verticillioides and for guidance in HPLC analysis of fumonisin accumulation in kernels. The work was supported by Specific Cooperative Agreement 58-6406-4-077 between United States Department of Agriculture-Agricultural Research Service and Texas A\&M University, the Texas Corn Producers Board, the Texas AgriLife Research Monocot Improvement program, and by National Science Foundation grants IOB-0544428 and AOS-0951272 to M. V. Kolomiets.

\section{LITERATURE CITED}

Bachmann, A., Hause, B., Maucher, H., Garbe, E., Vörös, K., Weichert, H., Wasternack, C. and Feussner, I. 2002. Jasmonate-induced lipid peroxidation in barley leaves initiated by distinct 13-LOX forms of chloroplasts. Biol. Chem. 383:1645-1657.

Bomblies, K. M., Wang, R. L., Ambrose, B. A., Schmidt, R. J., Meeley, R. B., and Doebley, J. 2003. Duplicate FLORICAULA/LEAFY homologs zfll and zfl2 control inflorescence architecture and flower patterning in maize. Development. 130:2385-2395.

Brenner, S., Johnson, M., Bridgham, J., Golda, G., Lloyd, D.H., Johnson, D., Luo, S., McCurdy, S., Foy, M., Ewan, M., Roth, R., George, D., Eletr, S., Albrecht, G., Vermaas, E., Williams, S.R., Moon, K., Burcham, T., Pallas, M., DuBridge, R.B., Kirchner, J., Fearon, K., Mao, J., and Corcoran, K. 2000. Gene expression analysis by massively parallel signature sequencing (MPSS) on microbead arrays. Nat. Biotechnol. 18:630-634.

Chenna, R., Sugawara, H., Koike, T., Lopez, R., Gibson, T. J., Higgins, D. G., and Thompson, J. D. 2003. Multiple sequence alignment with the Clustal series of programs. Nucleic Acids Res 31:3497-3500.

Christensen, S. A., and Kolomiets, M.V. 2011. The lipid language of plantfungal interactions. Fungal Genet. Biol. 48:4-14.

Christensen, S., Borrego, E., Shim, W. B., Isakeit, T., Kolomiets, M. 2012. Quantification of fungal colonization, sporogenesis, and production of mycotoxins using kernel bioassays. J. Vis. Exp. 62:e3727. Published online.

Christensen, S. A., Nemchenko, A., Borrego, E., Murray, I., Sobhy, I. S., Bosak, L., Deblasio, S., Erb, M., Robert, C. A., Vaughn, K. A., Herrfurth, C., Tumlinson, J., Feussner, I., Jackson, D., Turlings, T. C., Engelberth, J., Nansen, C., Meeley, R., and Kolomiets, M. V. 2013. The maize lipoxygenase, ZmLOX10, mediates green leaf volatile, jasmonate and herbivore-induced plant volatile production for defense against insect attack. Plant J. 74:59-73.

Colvin B. M, Harrison L. R. 1992. Fumonisin-induced pulmonary edema and hydrothorax in swine. Mycopathologia 117:79-82.

Constantino, N. N., Mastouri, F., Damarwinasis, R., Borrego, E. J., MoranDiez, M. E., Kenerley, C. M., Gao, X., and Kolomiets, M. V. 2013. Rootexpressed maize lipoxygenase 3 negatively regulates induced systemic resistance to Colletotrichum graminicola in shoots. Front. Plant Sci. 4:510.

De La Fuente G. N., Murray S. C., Isakeit T., Park Y. S., Yan Y., Warburton M. L., and Kolomiets M. V. 2013. Characterization of genetic diversity and linkage disequilibrium of $\mathrm{ZmLOX} 4$ and $\mathrm{ZmLOX5}$ loci in maize. PLoS One 8:e53973. doi: 10.1371/journal.pone.0053973. Published online.

Desjardins, A. E. 2003. Gibberella from A (venaceae) to Z (eae). Ann. Rev. Phytopathol. 41:177-198.

Desjardins, A. E., Harold W., Gardner I., and Klaus-M. 1992. Weltring Detoxification of sesquiterpene phytoalexins by Gibberella pulicaris (Fusarium sambucinum) and its importance for virulence on potato tubers. J. Ind. Microbiol. Biotechnol. 9:201-211

Dong, Q., Schlueter, S. D., and Brendel, V. 2004. PlantGDB, plant genome database and analysis tools. Nucleic Acids Res. 32:D354-D359.

Emrich, S. J., Aluru, S., Fu, Y., Wen T.-J., Narayanan, M., Guo, L., Ashlock D. A., and Schnable P. S. 2004. A strategy for assembling the maize
(Zea mays L.) genome. Bioinformatics 20:140-147.

Fammartino, A., Cardinale, F., Göbel, C., Mène-Saffrané, L., Fournier, J., Feussner, I., and Esquerré-Tugayé, M. T. 2007. Characterization of a divinyl ether biosynthetic pathway specifically associated with pathogenesis in tobacco. Plant Physiol. 143:378-88.

Feussner, I., and Kühn, H. 1995. The lipid body lipoxygenase from cucumber seedlings exhibits unusual reaction specificity. FEBS (Fed. Eur. Biochem. Soc.) Lett. 367:12-14.

Feussner, I., and Wasternack, C. 2002. The lipoxygenase pathway. Ann. Rev. Plant Biol. 53:275-297.

Feussner, I., Bachmann, A., Hohne, M., and Kindl, H. 1998. All three acyl moieties of trilinolein are efficiently oxygenated by recombinant Histagged lipid body lipoxygenase in vitro. FEBS (Fed. Eur. Biochem. Soc.) Lett. 431:433-436

Gao, X., Shim, W.-B., Göbel, C., Kunze, S., Feussner, I., Meeley, R., BalintKurti, P., and Kolomiets, M. 2007. Disruption of a maize 9-lipoxygenase results in increased resistance to fungal pathogens and reduced levels of contamination with mycotoxin fumonisin. Mol. Plant Microbe Interact. 20:922-933.

Gao, X., Starr, J., Göbel, C., Engelberth, J., Feussner, I., Tumlinson, J., and Kolomiets, M. 2008. Maize 9-lipoxygenase ZmLOX3 controls development, root-specific expression of defense genes, and resistance to root-knot nematodes. Mol. Plant Microbe Interact. 21:98-109.

Headley, J. V., Peru, K. M., Verma, B., and Robarts, R. D. 2002. Mass spectrometric determination of ergosterol in a prairie natural wetland. J. Chromatogr. A 958:149-156.

Heim, D., Nicholson, R. L., Pasholati, S. F., Hagerman, A. E., and Billet, W. 1983. Etiolated maize mesocotyls: A tool for investigating disease interactions. Phytopathology 73:424-428.

Hornung, E., Walther, M., Kühn, H., and Feussner, I. 1999. Conversion of cucumber linoleate 13-lipoxygenase to a 9-lipoxygenating species by site-directed mutagenesis. Proc. Natl. Acad. Sci. U.S.A. 96:41924197.

Howe, G., and Jander, G. 2008. Plant immunity to insect herbivores. Annu. Rev. Plant Biol. 59:41-66.

Hwang, I. S., and Hwang, B. K. 2010. The pepper 9-lipoxygenase gene CaLOX1 functions in defense and cell death responses to microbial pathogens. Plant Physiol. 152:948-967.

Isakeit, T., Gao, X., and Kolomiets, M. 2007. Exserohilum pedicellatum root rot of corn in Texas. Plant Dis. 91:634.

Kellerman, T. S., Marasas, W. F., Thiel, P. G., Gelderblom, W. C., Cawood, M., and Coetzer, J. A. 1990. Leukoencephalomalacia in two horses induced by oral dosing of fumonisin $\mathrm{B}_{1}$. Onderstepoort $\mathrm{J}$ Vet Res. 57:269-275.

Kidd, B. N., Edgar, C. I., Kumar, K. K., Aitken, E. A., Schenk, P. M., Manners, J. M., and Kazan, K. 2009. The mediator complex subunit PFT1 is a key regulator of jasmonate-dependent defense in Arabidopsis. Plant Cell 21:2237-2252.

Liavonchanka, A., and Feussner, I. 2006. Lipoxygenases: Occurrence, functions and catalysis. J. Plant Physiol. 163, 348-357.

Lopez, J. V., Kulasekaran, S., Vellosillo, T., Martinez, M., Irigoyen, M. L., Cascon, T., Bannenberg, G., Hamberg, M., and Castresana, C. 2011. Antagonistic role of 9-lipoxygenase-derived oxylipins and ethylene in the control of oxidative stress, lipid peroxidation and plant defense. The Plant J. 67:447-458

Makandar, R., Nalam, V., Chaturvedi, R., Jeannotte, R., Sparks, A. A., and Shah, J. 2010. Involvement of salicylate and jasmonate signaling pathways in Arabidopsis interaction with Fusarium graminearum. Mol. Plant-Microbe Interact. 23:861-870.

Marchler-Bauer, A., Anderson, J. B., Cherukuri, P. F., DeWeese-Scott, C., Geer, L. Y., Gwadz, M., He, S., Hurwitz, D. I., Jackson, J. D., Ke, Z., Lanczycki, C. J., Liebert, C. A., Liu, C., Lu, F., Marchler, G. H., Mullokandov, M., Shoemaker, B. A., Simonyan, V., Song, J. S., Thiessen, P. A., Yamashita, R. A., Yin, J. J., and Bryant, S. H. 2005. CDD: A conserved domain database for protein classification. Nucleic Acids Res. 33:D192-196.

McCarty, D., and Meeley, R. 2009. Transposon resources for forward and reverse genetics in maize. Pages 561-584 in: Handbook of Maize. Springer, New York.

Meeley, R., and Briggs, S. 1995. Reverse genetics for maize. Maize Newslett. 69:67-82.

Mueller, M. J., Mene-Saffrane, L., Grun, C., Karg, K., and Farmer, E. E. 2006. Oxylipin analysis methods. Plant J. 45:472-489.

Muller, M., and Munne-Bosch S. 2011. Rapid and sensitive hormone profiling of complex plant samples by liquid chromatograph coupled to electrospray ionization tandem mass spectrometry. Plant Methods 7:37. Published online.

Munkvold, G. P., McGee, D. C., and Carlton, W. M. 1997. Importance of different pathways for maize kernel infection by Fusarium moniliforme. Phytopathology 87:209-217. 
Nelson, P. E. 1992. Taxonomy and biology of Fusarium moniliforme. Mycopathologia 117:29-36.

Nemchenko, A., Kunze, S., Feussner, I., and Kolomiets, M. 2006. Duplicate maize 13-lipoxygenase genes are differentially regulated by circadian rhythm, cold stress, wounding, pathogen infection, and hormonal treatments. J. Exp. Bot. 57:3767-3779.

Oren, L., Ezrati, S., Cohen, D., and Sharon, A. 2003. Early events in the Fusarium verticillioides-maize interaction characterized by using a green fluorescent protein-expressing transgenic isolate. Appl. Environ. Microbiol. 69:1695-1701.

Prigge, S., Boyington, J., Gaffney, B., and Amzel, L. 1996. Structure conservation in lipoxygenases: structural analysis of soybean lipoxygenase-1 and modeling of human lipoxygenases. Proteins 24:275-291.

Prost, I., Dhondt, S., Rothe, G., Vicente, J., Rodriguez, M. J., Kift, N., Carbonne, F., Griffiths, G., Esquerre-Tugaye, M. T., Rosahl, S., Castresana, C., Hamberg, M., and Fournier, J. 2005. Evaluation of the antimicrobial activities of plant oxylipins supports their involvement in defense against pathogens. Plant Physiol. 139:1902-1913.

Rance, I. I., Fournier, J., and Esquerre-Tugaye, M. T. 1998. The incompatible interaction between Phytophthora parasitica var. nicotianae race 0 and tobacco is suppressed in transgenic plants expressing antisense lipoxygenase sequences. Proc. Natl. Acad. Sci. U.S.A. 95:6554 6559.

Rheeder, J. P., Marasas, W. F., and Vismer, H. F. 2002. Production of fumonisin analogs by Fusarium species. Appl. Environ. Microbiol. 68:2101-2105.

Santino, A., Iannacone, R., Hughes, R., Casey, R., and Mita, G. 2005. Cloning and characterisation of an almond 9-lipoxygenase expressed early during seed development. Plant Sci. 168:699-706.

Schmelz, E. A., Engelberth, J., Alborn, H. T., O’Donnell, P., Sammons, M., Toshima, H., and Tumlinson, J. H. 2003. Simultaneous analysis of phytohormones, phytotoxins, and volatile organic compounds in plants. Proc. Natl. Acad. Sci .U.S.A. 100:10552-10557.

Schmelz, E. A., Kaplan, F., Huffaker, A., Dafoe, N. J., Vaughan, M. M. Ni, X., Rocca, J. R., Alborn, H. T., and Teal, P. E. 2011. Identity, regulation, and activity of inducible diterpenoid phytoalexins in maize. Proc. Natl. Acad. Sci. U.S.A. 108:5455-60.
Shah, J. 2005. Lipids, lipases, and lipid-modifying enzymes in plant disease. Ann. Rev. Phytopathol 43:229-260.

Shim, W. B., and Woloshuk, C. P., 1999. Nitrogen repression of fumonisin $\mathrm{B}_{1}$ biosynthesis in Gibberella fujikuroi. FEMS (Fed. Eur. Microbiol. Soc.) Microbiol. Lett 177, 109-116.

Stintzi, A., Weber, H., Reymond P., Browse J. , and Farmer, E. E. 2001. Plant defense in the absence of jasmonic acid: The role of cyclopentenones. Proc. Natl. Acad. Sci. U.S.A. 98:12837-12842.

Sutton, G. S., White, O., Adams, M. D., and Kerlavage, A. R. 1995. TIGR assembler: A new tool for assemblying large shotgun sequencing projects. Genome Sci. Technol. 1:9-19.

Vicente, J., Cascón, T., Vicedo, B., García-Agustín, P., Hamberg, M., Castresana, C. 2012. Role of 9-lipoxygenase and a-dioxygenase oxylipin pathways as modulators of local and systemic defense. Mol. Plant 5:914-928.

Weber, H., Chételat, A., Caldelari, D., and Farmer, E. E. 1999. Divinyl ether fatty acid synthesis in late blight-diseased potato leaves. Plant Cell 11:485-493.

Yan, Y., Christensen, S., Isakeit, T., Engelberth, J., Meeley, R., Hayward, A., Emery, R. J., and Kolomiets, M. V. 2012 Disruption of OPR7 and OPR8 reveals the versatile functions of jasmonic acid in maize development and defense. Plant Cell 24:1420-1436.

Yoshizawa T., Yamashita A., and Luo Y. 1994. Fumonisin occurrence in corn from high- and low-risk areas for human esophageal cancer in China. Appl. Environ. Microbiol. 60:1626-1629.

Zhang, J., Simmons, C., Yalpani, N., Crane, V., Wilkinson, H., and Kolomiets M. 2005. Genomic analysis of the 12-oxo-phytodienoic acid reductase gene family of Zea mays. Plant Mol. Biol. 59:323-343.

Zhou, Y., Behrendt, J., Sutherland, A. J., and Griffiths G. 2011. Synthetic molecular mimics of naturally occurring cyclopentenones exhibit antifungal activity towards pathogenic fungi. Microbiology 157:3435-45.

\section{AUTHOR-RECOMMENDED INTERNET RESOURCES}

NCBI website: www.ncbi.nlm.nih.gov

Sorghum Genome Project website: www.phytozome.net/sorghum 